\title{
Telomerase in Bladder Cancer: Back to a Better Future?
}

\author{
Dan Theodorescu ${ }^{a, b, *}$, Thomas R. Cech ${ }^{b, c}$ \\ ${ }^{a}$ Department of Surgery, University of Colorado, Aurora, CO, USA; ${ }^{\mathrm{b}}$ University of Colorado Comprehensive Cancer Center, Aurora, CO, USA; ${ }^{\circ}$ Howard Hughes \\ Medical Institute, Department of Chemistry and Biochemistry, BioFrontiers Institute, University of Colorado, Boulder, CO, USA
}

Proper maintenance of chromosomes requires dealing with two biological challenges: the chromosome end-protection problem and the chromosome end-replication problem [1]. The first challenge stems from the need to distinguish the ends of linear chromosomes, which predominate in eukaryotes, from broken DNA ends that require repair. The second challenge stems from the gradual loss of sequence information at the extreme end of chromosomes, owing to incomplete replication by DNA polymerases (which only synthesize DNA in the $5^{\prime}$ to $3^{\prime}$ direction and are unable to fill in the gap left behind upon removal of the 5 -most RNA primer). Telomerase, a unique enzyme that contains telomerase reverse transcriptase (TERT) and a template-containing RNA component (telomerase RNA [TR]), extends telomeric DNA to solve both problems.

In mammals, the action of telomerase is required for the survival of continuously dividing cells such as embryonic cells and adult stem cells. These cells have relatively high levels of telomerase activity, whereas most somatic cells, which have a limited replicative lifespan, have very low or undetectable telomerase activity. Inactivation of telomerase in these latter cells is achieved mostly by transcriptional downregulation of the TERT gene, which encodes the essential, catalytically active protein subunit of the enzyme [2]. TERT protein associates with the essential TR subunit of the enzyme, which templates telomere extension, and with other protein factors to form the active holoenzyme that maintains telomere length [1].

TERT expression is also sometimes regulated by production of alternatively spliced messenger RNA (mRNA) variants that delete essential reverse transcriptase motifs of the enzyme. Increased abundance of these variants correlates with inactivation of telomerase during tissue differentiation, whereas increased levels of mRNAs encoding full-length TERT occur in some lymphomas and activated T cells [3-6]. Most cancer cells (85-90\%) upregulate TERT protein to reactivate telomerase and maintain telomere lengths through an indefinite number of cell divisions, and the remainder also maintain telomere length through an alternative lengthening of telomeres mechanism.

Recently, TERT promoter mutations have been found at high frequency in many diverse cancers but not in adjacent normal tissue $[7,8]$. An exciting breakthrough was finding point mutations in the TERT gene promoter in $66 \%$ of urothelial carcinomas of the bladder $[7,8]$. These mutations were first reported in melanoma $[9,10]$ and quickly found in other cancers $[7,8,10]$. In contrast, only $1 \%$ of cancers have mutations in the TERT open reading frame [11], and even these have not been shown to be activating mutations, suggesting that telomerase reactivation is primarily achieved through increased gene expression by transacting factors. Although these promoter mutations were hypothesized to increase expression of TERT by generating additional binding sites for E-twenty-six (ETS) transcription factors, only 1.15- to 2-fold increases in expression were found using a luciferase reporter gene system [9,10,12]. Additional mechanistic analysis is needed to determine if such modest increases in TERT expression can indeed drive immortalization or if the artificial reporter gene systems greatly underestimate the effect of the mutation; certainly other possibilities exist as well.

In this month's issue of European Urology, two studies $[13,14]$ follow up on these investigations and show that mutation analysis of the TERT promoter allows bladder tumor cells to be detected in urine. In addition, these studies show that TERT promoter mutations are found at similar

DOIs of original articles: http://dx.doi.org/10.1016/j.eururo.2013.08.052, http://dx.doi.org/10.1016/j.eururo.2013.08.057.

* Corresponding author. University of Colorado Cancer Center, Surgery, Cancer Research, 13001 E.17th Pl. MS \#F-434, Aurora, CO 80045, USA.

Tel. +1 303724 7135; Fax: +1 3037243162.

E-mail address: dan.theodorescu@ucdenver.edu (D. Theodorescu).

0302-2838/\$ - see back matter (C) 2013 Published by Elsevier B.V. on behalf of European Association of Urology.

http://dx.doi.org/10.1016/j.eururo.2013.10.019 
frequency across tumor stage, and there is lack of association with clinical outcome. These exciting studies further reinvigorate recent interest in telomerase in urothelial carcinoma that began in the early 1990s. In 1995, work indicated that in human urothelial carcinoma, telomere length was reduced in tumor tissue, more so in non-muscle-invasive than in invasive tumors, whereas telomerase was detectable only in tumor tissues and its activity was surprisingly unrelated to telomere length [15]. Other early work reported that telomerase activity had an association with the pathologic grade and clinical stage, with most advanced grade and deeply invasive tumors having high telomerase activity [16]. Many subsequent studies addressed whether telomerase could be used in some manner to detect bladder cancer [17], but none progressed to the clinical realm.

Taken together, these recent findings answer some questions and raise many others. It now appears that using current molecular technology, we can potentially detect approximately two-thirds of bladder cancers by searching for TERT promoter mutations in the urine. The clinical specificity, sensitivity, and positive and negative predictive values determined in larger prospective studies will be key in determining the value of any clinical test based on this observation. An important message also delivered by these studies is that TERT promoter mutations are not preferentially associated with either non-muscle-invasive or invasive bladder cancer and thus are likely to be a requirement for transformation or maintenance of malignancy in most urothelial tumors. Somewhat paradoxically, bladder cancers have shorter telomeres than cognate normal urothelium [15], also raising a question about the impact of these mutations. We do not yet understand the true biological contribution of these mutations to the malignant phenotype in urothelial carcinoma biology. Nevertheless, further work on this gene, its regulation, and its clinical utility will be very exciting, as it will likely lead to novel and practical insights into bladder cancer biology. In addition, we expect that these findings will rekindle interest in telomerase as an attractive therapeutic target.

Conflicts of interest: The authors have nothing to disclose.

Funding support: This work was supported in part by HHMI (to T.R.C.) and CA143971 (to D.T.).

\section{References}

[1] Nandakumar J, Cech TR. Finding the end: recruitment of telomerase to telomeres. Nat Rev Mol Cell Biol 2013;14:69-82.
[2] Lingner J, Hughes TR, Shevchenko A, Mann M, Lundblad V, Cech TR. Reverse transcriptase motifs in the catalytic subunit of telomerase. Science 1997;276:561-7.

[3] Jalink M, Ge Z, Liu C, Bjorkholm M, Gruber A, Xu D. Human normal T lymphocytes and lymphoid cell lines do express alternative splicing variants of human telomerase reverse transcriptase (hTERT) mRNA. Biochem Biophys Res Commun 2007;353:999-1003.

[4] Kilian A, Bowtell DD, Abud HE, et al. Isolation of a candidate human telomerase catalytic subunit gene, which reveals complex splicing patterns in different cell types. Hum Mol Genet 1997;6: 2011-9.

[5] Liu K, Hodes RJ, Weng N. Cutting edge: telomerase activation in human $\mathrm{T}$ lymphocytes does not require increase in telomerase reverse transcriptase (hTERT) protein but is associated with hTERT phosphorylation and nuclear translocation. J Immunol 2001;166: 4826-30.

[6] Ulaner GA, Hu JF, Vu TH, Giudice LC, Hoffman AR. Telomerase activity in human development is regulated by human telomerase reverse transcriptase (hTERT) transcription and by alternate splicing of hTERT transcripts. Cancer Res 1998;58:4168-72.

[7] Killela PJ, Reitman ZJ, Jiao Y, et al. TERT promoter mutations occur frequently in gliomas and a subset of tumors derived from cells with low rates of self-renewal. Proc Natl Acad Sci U S A 2013;110: 6021-6.

[8] Lotsch D, Ghanim B, Laaber M, et al. Prognostic significance of telomerase-associated parameters in glioblastoma: effect of patient age. Neuro Oncol 2013;15:423-32.

[9] Horn S, Figl A, Rachakonda PS, et al. TERT promoter mutations in familial and sporadic melanoma. Science 2013;339:959-61.

[10] Huang FW, Hodis E, Xu MJ, Kryukov GV, Chin L, Garraway LA. Highly recurrent TERT promoter mutations in human melanoma. Science 2013;339:957-9.

[11] The cancer genome atlas. National Institutes of Health Web site. http://cancergenome.nih.gov.

[12] Xu D, Dwyer J, Li H, Duan W, Liu JP. Ets2 maintains hTERT gene expression and breast cancer cell proliferation by interacting with c-Myc. J Biol Chem 2008;283:23567-80.

[13] Allory Y, Beukers W, Sagrera A, et al. Telomerase reverse transcriptase promoter mutations in bladder cancer: high frequency across stages, detection in urine, and lack of association with outcome. Eur Urol 2014;65:360-6.

[14] Hurst CD, Platt FM, Knowles MA. Comprehensive mutation analysis of the TERT promoter in bladder cancer and detection of mutations in voided urine. Eur Urol 2014;65:367-9.

[15] Kamata S, Kageyama Y, Yonese J, Oshima H. Significant telomere reduction in human superficial transitional cell carcinoma. Br J Urol 1996;78:704-8

[16] Lin Y, Miyamoto H, Fujinami K, et al. Telomerase activity in human bladder cancer. Clin Cancer Res 1996;2:929-32.

[17] Dalbagni G, Han W, Zhang ZF, et al. Evaluation of the telomeric repeat amplification protocol (TRAP) assay for telomerase as a diagnostic modality in recurrent bladder cancer. Clin Cancer Res 1997;3: 1593-8. 